\title{
Change detection in polarimetric SAR data and the complex Wishart distribution
}

\author{
Conradsen, Knut; Nielsen, Allan Aasbjerg; Schou, Jesper; Skriver, Henning
}

Published in:

Proceedings of the IEEE 2001 International Geoscience and Remote Sensing Symposium

Link to article, DOI:

10.1109/IGARSS.2001.978111

Publication date:

2001

Document Version

Publisher's PDF, also known as Version of record

Link back to DTU Orbit

Citation (APA):

Conradsen, K., Nielsen, A. A., Schou, J., \& Skriver, H. (2001). Change detection in polarimetric SAR data and the complex Wishart distribution. In Proceedings of the IEEE 2001 International Geoscience and Remote Sensing Symposium (Vol. 6) https://doi.org/10.1109/IGARSS.2001.978111

\section{General rights}

Copyright and moral rights for the publications made accessible in the public portal are retained by the authors and/or other copyright owners and it is a condition of accessing publications that users recognise and abide by the legal requirements associated with these rights.

- Users may download and print one copy of any publication from the public portal for the purpose of private study or research.

- You may not further distribute the material or use it for any profit-making activity or commercial gain

- You may freely distribute the URL identifying the publication in the public portal 


\title{
Change Detection in Polarimetric SAR Data and the Complex Wishart Distribution
}

\author{
Knut Conradsen ${ }^{1}$, Allan Aasbjerg Nielsen ${ }^{1}$, Jesper Schou $^{2}$ and Henning Skriver ${ }^{2}$ \\ ${ }^{1}$ IMM, Informatics and Mathematical Modelling, Building 321 \\ ${ }^{2}$ EMI, Section for Electromagnetic Systems, Ørsted•DTU, Building 348 \\ Technical University of Denmark, DK-2800 Kgs. Lyngby, Denmark \\ e-mail aa@imm.dtu.dk, internet www.imm.dtu.dk/ aa
}

\begin{abstract}
When working with multi-look fully polarimetric synthetic aperture radar (SAR) data an appropriate way of representing the backscattered signal consists of the so-called covariance matrix. For each pixel this is a $3 \times 3$ Hermitian, positive definite matrix which follows a complex Wishart distribution. Based on this distribution a test statistic for equality of two such matrices and an associated asymptotic probability for obtaining a smaller value of the test statistic are given and applied to change detection in polarimetric SAR data. In a case study EMISAR $L$ band data from 17 April 1998 and 20 May 1998 covering agricultural fields near Foulum, Denmark are used. The derived test statistic can be applied as a line or edge detector in fully polarimetric SAR data also.

Index Terms-Covariance matrix test statistic, radar polarimetry, radar applications, remote sensing change detection, EMISAR.
\end{abstract}

\section{INTRODUCTION}

$\mathrm{D}$ UE TO ITS all-weather mapping capability synthetic aperture radar (SAR) data holds a strong potential for e.g. change detection studies in remote sensing applications. In this paper multitemporal SAR mages of agricultural fields are used to demonstrate a new change detection method for polarimetric SAR data. It is well-known that the development of different crops over time causes changes in the backscatter. The radar backscattering is sensitive to the dielectric properties of the vegetation and the soil, to the plant structure (i.e., the size, shape, and orientation distributions of the scatterers), to the surface roughness, and to the canopy structure (e.g., row direction and spacing, and cover fraction) [1], [2].

The polarimetric SAR measures the amplitude and phase of backscattered signals in four combinations of the linear receive and transmit polarizations: $\mathrm{HH}, \mathrm{HV}, \mathrm{VH}$, and VV. These signals form the complex scattering matrix which relates the scattered to the incident electric fields [3]. The inherent speckle in the SAR data can be reduced by spatial averaging at the expense of loss of spatial resolution. In this so-called multi-look case a more appropriate representation of the backscattered signal is the covariance matrix in which the average properties of a group of resolution cells can be expressed in a single matrix. The average covariance matrix is defined as [3]

$$
\langle\boldsymbol{C}\rangle=\left[\begin{array}{lll}
\left\langle S_{h h} S_{h h}^{*}\right\rangle & \left\langle S_{h h} S_{h v}^{*}\right\rangle & \left\langle S_{h h} S_{v v}^{*}\right\rangle \\
\left\langle S_{h v} S_{h h}^{*}\right\rangle & \left\langle S_{h v} S_{h v}^{*}\right\rangle & \left\langle S_{h v} S_{v v}^{*}\right\rangle \\
\left\langle S_{v v} S_{h h}^{*}\right\rangle & \left\langle S_{v v} S_{h v}^{*}\right\rangle & \left\langle S_{v v} S_{v v}^{*}\right\rangle
\end{array}\right]
$$

where $\langle\cdot\rangle$ denotes ensemble averaging, ${ }^{*}$ denotes complex conjugation, and $S_{r t}$ is the complex scattering amplitude for receive polarization $r$ and transmit polarization $t$ ( $r$ and $t$ are either $h$ for horizontal or $v$ for vertical). Reciprocity, which normally applies to natural targets, gives $S_{h v}=S_{v h}$ (in the backscattering direction using the backscattering alignment convention [3]) and results in the covariance matrix (1) with rank 3. $\langle\boldsymbol{C}\rangle$ follows a complex Wishart distribution.

In this article a test statistic for equality of two complex covariance matrices and an associated asymptotic probability measure for obtaining a smaller value of the test statistic are given and applied to change detection in fully polarimetric SAR data. In [4] a change detection scheme based on canonical correlations analysis is applied to scalar EMISAR data, see also [5], [6].

If used with $\mathrm{HH}, \mathrm{VV}$ or $\mathrm{HV}$ data only the test statistic reduces to the well-known test statistic for equality of the scale parameters in two gamma distributions.

For a more thorough description of the method, see [7]. The given test statistic can be applied as a line or edge detector in fully polarimetric SAR data also, [8], [9].

\section{THEORY}

This section very briefly describes the complex normal and Wishart distributions, and the likelihood ratio test for equality of two complex Wishart matrices. For a more thorough description, see [7] and references therein.

\section{A. The Complex Normal Distribution}

We say that a $p$-dimensional random complex vector $\boldsymbol{Z}$ follows a complex multivariate normal distribution with mean $\mathbf{0}$ and dispersion matrix $\boldsymbol{\Sigma}$, i.e.,

$$
\boldsymbol{Z}=\left[\begin{array}{lll}
Z_{1} & \cdots & Z_{p}
\end{array}\right]^{T} \in N_{C}(\mathbf{0}, \boldsymbol{\Sigma})
$$

if the frequency function is

$$
\begin{aligned}
f(\boldsymbol{z}) & =\frac{1}{\pi^{p}|\boldsymbol{\Sigma}|} \exp \left\{-\boldsymbol{z}^{* T} \boldsymbol{\Sigma}^{-1} \boldsymbol{z}\right\} \\
& =\frac{1}{\pi^{p}|\boldsymbol{\Sigma}|} \exp \left\{-\operatorname{tr}\left[\boldsymbol{\Sigma}^{-1} \boldsymbol{z} \boldsymbol{z}^{* T}\right]\right\}
\end{aligned}
$$

where $|\cdot|$ denotes the determinant, tr denotes the trace of a matrix, and ${ }^{* T}$ denotes complex conjugation $\left(^{*}\right)$ and transpose $\left({ }^{T}\right)$.

\section{B. The Complex Wishart Distribution}

We say that a Hermitian, positive definite random $p \times p$ matrix $\boldsymbol{W}$ follows a complex Wishart distribution, i.e.,

$$
\boldsymbol{W} \in W_{C}(p, n, \boldsymbol{\Sigma})
$$


if the frequency function is

$$
f(\boldsymbol{w})=\frac{1}{\Gamma_{p}(n)} \frac{1}{|\boldsymbol{\Sigma}|^{n}}|\boldsymbol{w}|^{n-p} \exp \left\{-\operatorname{tr}\left[\boldsymbol{\Sigma}^{-1} \boldsymbol{w}\right]\right\}
$$

where

$$
\Gamma_{p}(n)=\pi^{p(p-1) / 2} \prod_{j=1}^{p} \Gamma(n-j+1) .
$$

The frequency function is defined for $\boldsymbol{w}$ positive definite.

If $\boldsymbol{X}$ and $\boldsymbol{Y}$ are independent and both follow complex Wishart distributions,

$$
\boldsymbol{X} \in W_{C}(p, n, \boldsymbol{\Sigma}) \text { and } \boldsymbol{Y} \in W_{C}(p, m, \boldsymbol{\Sigma}),
$$

then their sum also follows a complex Wishart distribution,

$$
\boldsymbol{S}=\boldsymbol{X}+\boldsymbol{Y} \in W_{C}(p, n+m, \boldsymbol{\Sigma}) .
$$

\section{Test for Equality of Two Complex Wishart Matrices}

Let the independent $p \times p$ Hermitian, positive definite matrices $\boldsymbol{X}$ and $\boldsymbol{Y}$ be complex Wishart distributed, i.e., $\boldsymbol{X} \in$ $W_{C}\left(p, n, \boldsymbol{\Sigma}_{x}\right)$ with $\hat{\boldsymbol{\Sigma}}_{x}=\frac{1}{n} \boldsymbol{X}$ and $\boldsymbol{Y} \in W_{C}\left(p, m, \boldsymbol{\Sigma}_{y}\right)$ with $\hat{\boldsymbol{\Sigma}}_{y}=\frac{1}{m} \boldsymbol{Y}$. We consider the null hypothesis $H_{0}: \boldsymbol{\Sigma}_{x}=\boldsymbol{\Sigma}_{y}$ which states that the two matrices are equal against the alternative hypothesis $H_{1}: \boldsymbol{\Sigma}_{x} \neq \boldsymbol{\Sigma}_{y}$.

If $H_{0}$ is true (in statistical parlance: "under $H_{0}$ ") $\boldsymbol{S}=\boldsymbol{X}+$ $\boldsymbol{Y} \in W_{C}(p, n+m, \boldsymbol{\Sigma})$ with $\hat{\boldsymbol{\Sigma}}=\frac{1}{n+m} \boldsymbol{S}=\frac{1}{n+m}(\boldsymbol{X}+\boldsymbol{Y})$. The likelihood ratio test statistic becomes

$$
Q=\frac{L(\hat{\boldsymbol{\Sigma}})}{L_{x}\left(\hat{\boldsymbol{\Sigma}}_{x}\right) L_{y}\left(\hat{\boldsymbol{\Sigma}}_{y}\right)} .
$$

Here

$$
L_{x}\left(\hat{\boldsymbol{\Sigma}}_{x}\right)=\frac{1}{\Gamma_{p}(n)}\left|\frac{1}{n} \boldsymbol{X}\right|^{-n}|\boldsymbol{X}|^{n-p} \exp \{-n \operatorname{tr} \boldsymbol{I}\}
$$

(and similarly for $L_{y}\left(\hat{\boldsymbol{\Sigma}}_{y}\right)$ ) where $\boldsymbol{I}$ is the identity matrix (tr $\boldsymbol{I}=$ $p$ ). For the numerator of $Q$ we get

$$
\begin{aligned}
L(\hat{\boldsymbol{\Sigma}})= & \frac{1}{\Gamma_{p}(n) \Gamma_{p}(m)}\left|\frac{1}{n+m}(\boldsymbol{X}+\boldsymbol{Y})\right|^{-(n+m)} \times \\
& |\boldsymbol{X}|^{n-p}|\boldsymbol{Y}|^{m-p} \exp \{-(n+m) \operatorname{tr} \boldsymbol{I}\}
\end{aligned}
$$

This leads to the desired likelihood ratio test statistic

$$
Q=\frac{(n+m)^{p(n+m)}}{n^{p n} m^{p m}} \frac{|\boldsymbol{X}|^{n}|\boldsymbol{Y}|^{m}}{|\boldsymbol{X}+\boldsymbol{Y}|^{n+m}} .
$$

If $n=m$ which is typically the case for change detection we get

$$
\ln Q=n(2 p \ln 2+\ln |\boldsymbol{X}|+\ln |\boldsymbol{Y}|-2 \ln |\boldsymbol{X}+\boldsymbol{Y}|) .
$$

With

$$
\begin{gathered}
\rho=1-\frac{2 p^{2}-1}{6 p}\left(\frac{1}{n}+\frac{1}{m}-\frac{1}{n+m}\right) \quad \text { and } \\
\omega_{2}=-\frac{p^{2}}{4}\left(1-\frac{1}{\rho}\right)^{2}+\frac{p^{2}\left(p^{2}-1\right)}{24 \rho^{2}}\left(\frac{1}{n^{2}}+\frac{1}{m^{2}}-\frac{1}{(n+m)^{2}}\right)
\end{gathered}
$$

the probability of finding a smaller value of $-2 \rho \ln Q$ is

$$
\begin{aligned}
& P\{-2 \rho \ln Q \leq z\} \simeq P\left\{\chi^{2}\left(p^{2}\right) \leq z\right\}+ \\
& \omega_{2}\left[P\left\{\chi^{2}\left(p^{2}+4\right) \leq z\right\}-P\left\{\chi^{2}\left(p^{2}\right) \leq z\right\}\right] .
\end{aligned}
$$

For covariance matrix data $p=3$. For $\mathrm{HH}, \mathrm{HV}$ or $\mathrm{VV}$ data $p=1$. In the latter case $\boldsymbol{X}$ and $\boldsymbol{Y}$ are therefore scalars $X$ and $Y$, and $Q$ reduces to

$$
Q=\frac{(n+m)^{n+m}}{n^{n} m^{m}} \frac{X^{n} Y^{m}}{(X+Y)^{n+m}}
$$

which is equivalent to the well-known likelihood ratio test statistic for the equality of two gamma parameters [10], [11].

For space limitation reasons the important case with azimuthal symmetry is not treated here, see [7].

\section{CASE STUdy: EMISAR}

To illustrate the change detection capability of the derived test statistic this case study uses EMISAR from an agricultural test site at the Research Center Foulum located in Central Jutland, Denmark. The data used here are part of the data used in a previously reported study on polarimetric signatures of crops [2].

The EMISAR is the result of a research and development project initiated in 1986 at the Department of Electromagnetic Systems (now Oersted-DTU Department) of the Technical University of Denmark, [12], [13]. The EMISAR system is fully polarimetric and interferometric and operates at two frequencies, $L$-band (1.25 GHz/25 cm wavelength) and $C$-band (5.3 GHz/5.7 $\mathrm{cm}$ wavelength). The EMISAR is flown on a Royal Danish Airforce Gulfstream G-3 aircraft and is normally operated from an altitude of approximately $12,500 \mathrm{~m}$, the spatial resolution is $2 \times 2$ $\mathrm{m}^{2}$ (one-look), the ground range swath is approximately $12 \mathrm{~km}$, and typical incidence angles range from $35^{\circ}$ to $60^{\circ}$. The processed data from this system are fully calibrated by means of an advanced internal calibration system. Well-calibrated data are very important in the change detection scheme set up in this article. Two EMISAR $L$-band images from 17 April 1998 and 20 May 1998, respectively, of the test site at Foulum are shown in Figs. 1 and 2 as color composites of the HV (actually the complex addition of HV and VH; red), HH (green), and VV (blue) channels. The single look scattering matrix data has been converted to covariance matrix data, and at the same time speckle reduced by a raised cosine filter to an equivalent number of looks of approximately 11 . The geometrical co-registration is however very important in a change detection application, where two images are compared on a pixel-by-pixel basis. The polarimetric images were registered to a digital elevation model generated from interferometric data acquired by EMISAR. The registration was carried out by combining a model of the imaging geometry with few ground control points, and the images were registered to one another with an rms-accuracy of better than one pixel [14].

\section{RESUlTS AND DisCUSSION}

Figs. 3 and 4 show $\ln Q$ and the probability of finding a smaller value of $-2 \rho \ln Q$ for full covariance matrix data. Dark regions in Fig. 3 and bright regions in Fig. 4 are regions of high change. The bright areas primarily in the lower half of Figs. 1 
and 2 are coniferous forests and we observe very small change for these areas (Figs. 3 and 4), due to the very stable backscatter from such forest areas (see e.g. [15]). The agricultural fields show different degrees of change, dependent on the crop type. In the upper left corner three small fields show change, no-change and change behavior, respectively. The field with no change is a beet field, which for both acquisitions is a bare field. The two other fields are spring barley fields, which are bare at the first acquisition and have a $12-14 \mathrm{~cm}$ vegetation layer at the second acquisition. The three large fields at the right part of the image are from left to right: grass, winter wheat and spring barley. The spring barley field shows even larger change behavior than the smaller fields to the left. The grass field is virtually unchanged between the two acquisitions. The vegetation height for the winter wheat field has changed from 14 to $42 \mathrm{~cm}$ between the two acquisitions, and we observe a moderate change between the images. It is obvious from the results presented here and the more detailed evaluation in [7] that the new change detection method is working very well when changes in the polarimetric parameters are experienced.

\section{REFERENCES}

[1] F. T. Ulaby, R. K. Moore, and A. K. Fung, Microwave Remote Sensing. Active and Passive, Artech, Dedham, MA, 1986, vol. 3.

[2] H. Skriver, M. T. Svendsen, and A. G. Thomsen, "Multitemporal $L$ - and $C$-band polarimetric signatures of crops," IEEE Transactions on Geoscience and Remote Sensing, vol. 37, pp. 2413-2429, 1999.

[3] J. J. van Zyl and F. T. Ulaby, "Scattering matrix representation for simple targets," in Radar Polarimetry for Geoscience Applications, F. T. Ulaby and C. Elachi, Eds. Artech, Norwood, MA, 1990.

[4] A. A. Nielsen, R. Larsen, and H. Skriver, "Change detection in bi-tempora EMISAR data from Kalø, Denmark, by means of canonical correlation analysis," in Proceedings of the Third International Airborne Remote Sensing Conference and Exhibition, ERIM, Ed., Copenhagen, Denmark, 7-10 July 1997.

[5] A. A. Nielsen, K. Conradsen, and J. J. Simpson, "Multivariate alteration detection (MAD) and MAF post-processing in multispectral, bi-tempora image data: New approaches to change detection studies," Remote Sensing of Environment, vol. 19, pp. 1-19, 1998.

[6] A. A. Nielsen, "Multi-channel remote sensing data and orthogonal transformations for change detection," in Machine Vision and Advanced Image Processing in Remote Sensing, I. Kanellopoulos, G. G. Wilkinson, and T. Moons, Eds., pp. 37-48. Springer, 1999.

[7] K. Conradsen, A. A. Nielsen, J. Schou, and H. Skriver, "A test statistic in the complex Wishart distribution and its application to change detection in polarimetric SAR data," unpublished, 2001.

[8] J. Schou, H. Skriver, K. Conradsen, and A. A. Nielsen, "CFAR edge detector for polarimetric SAR data," unpublished, 2001.

[9] H. Skriver, J. Schou, K. Conradsen, and A. A. Nielsen, "Polarimetric edge detector based on the complex Wishart distribution," in these proceedings, 2001.

[10] R. Touzi, A. Lopes, and P. Bousquez, "A statistical and geometrical edge detector for SAR images," IEEE Transactions on Geoscience and Remote Sensing, vol. 26, no. 6, pp. 764-773, Nov. 1988.

[11] A. Lopes, E. Nezry, R. Touzi, and H. Laur, "Structure detection and statistical adaptive speckle filtering in SAR images," International Journal of Remote Sensing, vol. 13, no. 9, pp. 1735-1758, 1993.

[12] S. N. Madsen, E. L. Christensen, N. Skou, and J. Dall, "The Danish SAR system: Design and initial tests," IEEE Transactions on Geoscience and Remote Sensing, vol. 29, pp. 417-476, 1991.

[13] E. L. Christensen, N. Skou, J. Dall, K. Woelders, J. H. Jørgensen, J. Granholm, and S. N. Madsen, "EMISAR: An absolutely calibrated polarimetric $L$ - and $C$-band SAR," IEEE Transactions on Geoscience and Remote Sensing, vol. 36, pp. 1852-1865, 1998.

[14] W. Dierking, J. Schou, and H. Skriver, "Change detection of small objects and linear features in multi-temporal polarimetric images," in Proceedings of the International Geoscience and Remote Sensing Symposium (IGARSS) 2000, IEEE, Ed., Honolulu, Hawaii, USA, 24-28 July 2000, pp. $1693-$ 1695.

[15] W. Dierking and H. Skriver, "Change detection for topographic mapping by menas of airborne multi-temporal polarimetric SAR imagery," 2001.

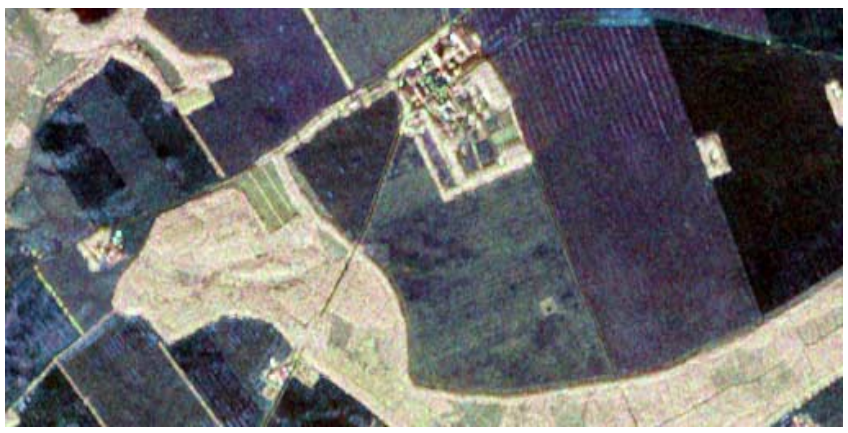

Fig. 1. $L$-band EMISAR data from 17 April 1998

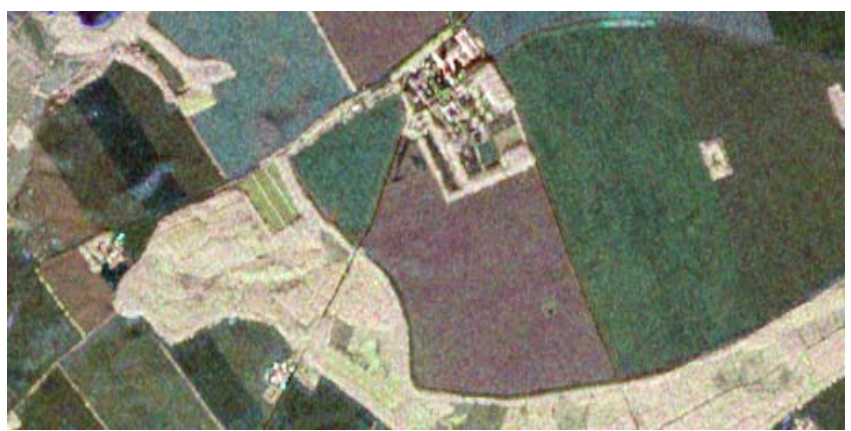

Fig. 2. $L$-band EMISAR data from 20 May 1998

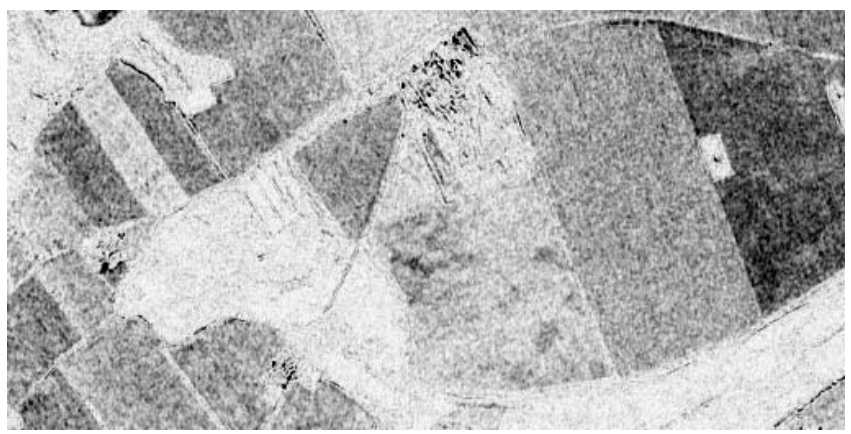

Fig. 3. $L$-band EMISAR change, $\ln Q$, full covariance matrix

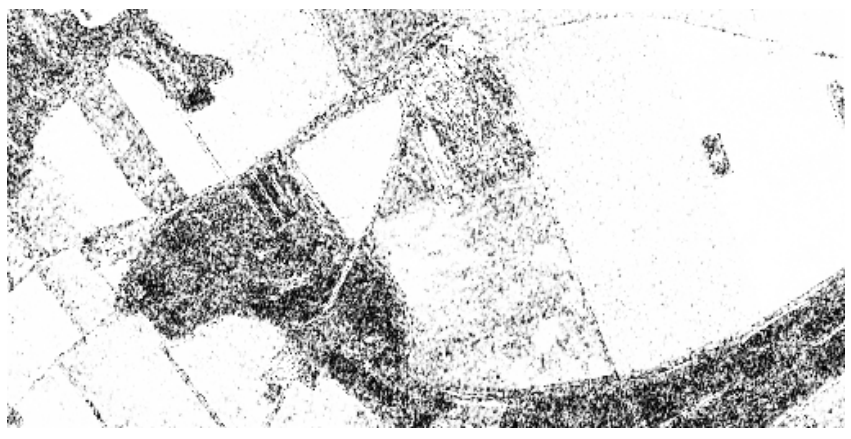

Fig. 4. $L$-band EMISAR change, $P\{-2 \rho \ln Q \leq z\}$, full covariance matrix 